\title{
Haines jumps: Pore scale mechanisms
}

\author{
Zhonghao Sun* and J. Carlos Santamarina \\ Earth Science and Engineering, King Abdullah University of Science and Technology (KAUST), Thuwal 23955-6900, Saudi Arabia
}

(Received 24 December 2018; published 27 August 2019)

\begin{abstract}
Haines instabilities are sudden jumps of the fluid interface accompanied by fluid redistribution and a transient pressure response. Haines jumps affect global displacement patterns in porous media, promote fingered invasion, increase fluid trapping, and contribute to hysteretic saturation. Detailed analyses and experimental results show that jumps take place when the pressure-volume response is multivalued across the pore throat, during either advancing or receding tests. This situation emerges in "soft systems" such as liquids with entrapped gas bubbles, compliant substrates, and when multiple menisci interact across the porous networks. Elastic deformations and capillarity combine to form the dimensionless elastocapillary number $N_{\mathrm{ec}}$ for a given pore geometry and fluid mixture: a system with elastocapillary number $N_{\mathrm{ec}}<1$ is prone to Haines instabilities. Therefore, Haines jumps are more likely to occur not only in soft porous systems, but also when the network topology is characterized by pronounced pore constrictions, and the fluids form small contact angles and generate high interfacial tension.
\end{abstract}

DOI: 10.1103/PhysRevE.100.023115

\section{INTRODUCTION}

Capillarity determines immiscible flow in porous media [1]. Haines instabilities describe sudden jumps of the fluid interface accompanied by fluid redistribution and a transient pressure response [2] [Figs. 1(a) and 1(b)]. Haines jumps are ubiquitous in porous media. They cause unstable local displacement, increase fluid discontinuity, and promote fingered invasion $[3,4]$. As a result, Haines instabilities affect global displacement patterns, increase fluid trapping, and contribute to hysteretic saturation in porous media [5-7].

Early studies used descriptive terms such as "unstable point" and "unstable interface" without a specified criterion for Haines jumps [2,3]. A local thermodynamic analysis suggests that an interface configuration is stable when $d J / d V_{w}<$ 0 , where the meniscus curvature $J$ is defined by the principal radii $J=1 / r_{1}+1 / r_{2}$, and $V_{w}$ is the volume of the wetting phase [8-12]. However, this criterion contradicts experimental observations of liquid penetration into periodically constricted capillaries $[13,14]$ and experiments conducted with careful volume-controlled displacement [15]. Furthermore, recent studies also show that a Haines jump at one meniscus affects capillary conditions at nearby interconnected menisci $[16,17]$, and flow complexity is aggravated by the presence of surfactants and/or contaminants $[15,18]$.

This paper presents a detailed analytical and experimental study of pore scale mechanisms that underlie Haines jumps in porous media. We consider pure fluids without surfactants or contaminants. Our goals are to understand causal links and to identify governing parameters in view of the potential control and manipulation of this instability.

\section{CONCEPTUAL FRAMEWORK}

Consider the displacement of a wetting liquid (e.g., water) by a nonwetting fluid (e.g., air or oil) across a pore

*zsun@utexas.edu constriction, and ignore the effects of viscosity and gravity as both the capillary number and Bond number are small (note: in this study, the characteristic length for the capillary number is the pore scale and the effect of viscosity is secondary-see $[19,20]$ for more discussion). The pressure difference $\Delta P(x)$ between the wetting and the non-wetting fluids is a function of the surface tension $\gamma$, the contact angle $\theta$, and the pore geometry [15]:

$$
\Delta P(x)=P_{w}-P_{n}=-\frac{4 \gamma \cos [\theta-\alpha(x)]}{d(x)},
$$

where $\alpha(x)$ is the local angle between the pore wall surface and the flow direction, and $d(x)$ is the pore diameter at position $x$ [Fig. 2(a)]. For convenience, let us compute the wetting fluid volume from the reference meniscus at position $x=0$ (the center of the constriction) and assume a constant fluid pressure in the non-wetting phase $P_{n}=P_{0}$; then $\Delta P(x)$ is the differential pressure in the wetting liquid relative to $P_{0}$ (negative value of the capillary pressure).

Figure 2(b) shows the computed pressure-volume signature $\Delta P-V_{\text {pore }}$ for the sinusoidal pore (length $L$, opening diameter $H H^{\prime}=2 r_{1}$, and constriction diameter $A A^{\prime}=2 r_{2}$ ). The pressure decreases as the meniscus moves towards the constriction, reaches a minimum near the pore throat, and then increases as the meniscus moves away from the constriction. Note that there is a one-to-one correspondence between pressure $\Delta P$ and volume $V_{\text {pore }}$.

The single pore analyzed above is part of a porous network. In natural or engineered porous systems, the pore liquid may not be incompressible and may have entrapped gas bubbles, the solid matrix may not be perfectly rigid, and there are nearby interconnected menisci. These conditions create a "soft system" and pressure changes in the pore liquid cause an elastic volumetric change $V_{\mathrm{el}}$ in the associated system [Fig. 2(c)].

The actual system response $\Delta P-V_{\text {sys }}$ during liquid invasion combines the invaded pore volume $V_{\text {pore }}$ with the system's 
(a)

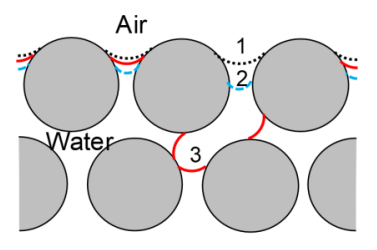

(b)

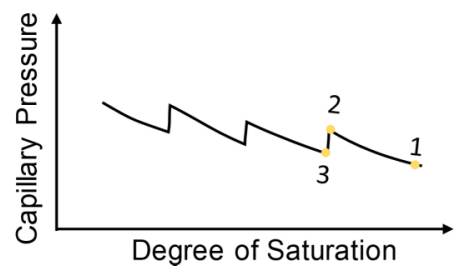

FIG. 1. Haines jumps during drying in porous media. (a) Interfacial configuration. (b) Transient capillary pressure response.

elastic volumetric change $V_{\mathrm{el}}$ :

$$
V_{\text {sys }}=V_{\text {pore }}-V_{\mathrm{el}} \text {. }
$$

The resultant $\Delta P$ - $V_{\text {sys }}$ curve [Fig. 2(d)] can be multivalued across the pore throat and a jump will take place during either advancing or receding tests. Let us compute the system pressure-volume relationship for the three soft pore models sketched in Fig. 3.

\section{A. Mechanism 1: Soft liquid-entrapped gas bubble}

Gas bubbles entrapped in the liquid expand or shrink in response to pressure changes [Fig. 3(b)]. Assume the gas bubble volume is $V_{0}$ when the meniscus is at the reference position $x=0$. As the meniscus moves to position $x$, the bubble volume changes in response to the pressure change in

(a) Pore constriction geometry

(b) Fluid displacement
$\Delta \mathrm{P}-\mathrm{V}_{\text {pore }}$

(b) Fluid displacement
$\Delta \mathrm{P}-\mathrm{V}_{\text {pore }}$
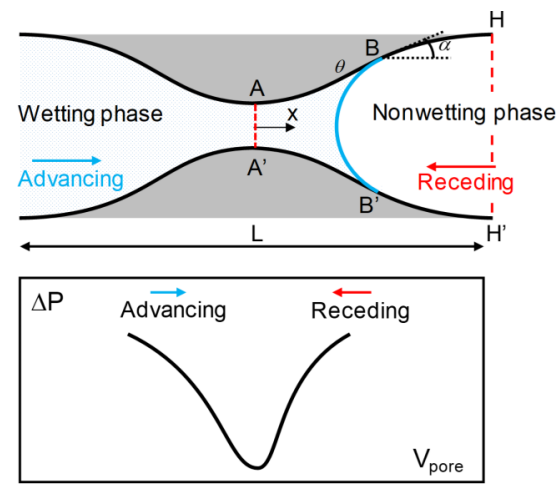

(c) System compliance $\Delta \mathrm{P}-\mathrm{V}_{\mathrm{el}}$

(d) Measured response $\Delta \mathrm{P}-\mathrm{V}_{\text {sys }}$
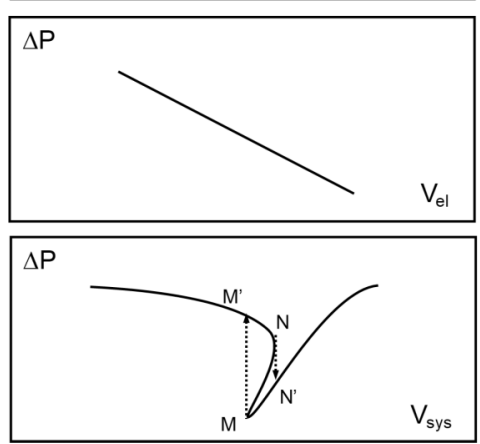

FIG. 2. Conceptual framework: Haines jumps are consequences of elastic and capillary interactions. (a) A sinusoidal pore constriction. (b) Pressure-volume signature $\Delta P-V_{\text {pore }}$ in a single rigid pore, where $\Delta P=P_{w}-P_{n}$, as defined in Eq. (1). (c) System compliance response $\Delta P-V_{\mathrm{el}}$. (d) Combined pressure-volume signature $\Delta P-V_{\text {sys }}$. The multivalued response shows Haines jumps at turning points $\mathrm{M}, \mathrm{N}$.

the pore liquid (assumptions: ideal gas law, disregards surface tension as a first approximation):

$$
V_{\mathrm{el}}(x)=\left[\frac{P_{0}+\Delta P(0)}{P_{0}+\Delta P(x)}-1\right] V_{0} .
$$

Then, the injected liquid volume $V_{\text {sys }}$ that is required to advance the interface to position $x$ is $V_{\text {sys }}=V_{\text {pore }}-V_{\text {el }}$ [Eq. (2)].

Figures 3(a) and 3(b) show the pressure-volume response when a wetting liquid recedes across a sinusoidal pore constriction ( $L=4 \mathrm{~mm}, r_{1}=0.5 \mathrm{~mm}$, and $r_{2}=0.2 \mathrm{~mm}$ ). Figure 3(a) shows the $\Delta P-V_{\text {sys }}$ relationship for the case without a gas bubble $\left(V_{0}=0\right)$; this trend is also the $\Delta P-V_{\text {pore }}$ relationship for the stiff system $V_{\mathrm{el}}=0$. The dashed line in Fig. 3(b) shows the elastic response $\Delta P-V_{\mathrm{el}}$ for the case of a small gas bubble $\left(V_{0}=0.1 \mathrm{ml}\right)$. The combined system $\Delta P-V_{\mathrm{sys}}$ curve becomes multivalued (solid blue line). Therefore, the meniscus jumps from point $\mathrm{M}$ to a new equilibrium position $\mathrm{M}^{\prime}$ in the upper branch during the receding test [the inset in Fig. 3(b) enlarges the $\Delta P-V_{\text {sys }}$ response around point $\left.\mathrm{M}\right]$. A similar analysis explains drop formation instabilities as well [21].

\section{B. Mechanism 2: Deformable solid matrix}

The solid matrix is not perfectly rigid in natural formations (e.g., poroelasticity) or laboratory systems [e.g., deformable plastic tubes and polydimethylsiloxane (PDMS) micromodels]. Therefore, the pore volume changes in response to the evolving capillary pressure. Let us assume that the volumetric response of the solid matrix has an elastic stiffness $k\left(\mathrm{~Pa} / \mathrm{m}^{3}\right)$; then the pore volume reduction $V_{\mathrm{el}}$ when the meniscus moves to position $x$ is

$$
V_{\mathrm{el}}=\frac{-[\Delta P(x)-\Delta P(0)]}{k} .
$$

For example, an elastic tube of length $L_{\text {tube }}$, inner diameter $a$, outer diameter $b$, and shear modulus $G$ has an elastic stiffness $k$ equal to [22] (assume Poisson ratio $v=0.5$ )

$$
k=G\left(\frac{1}{a^{2}}-\frac{1}{b^{2}}\right) \frac{1}{\pi L_{\text {tube }}} .
$$

Figure 3(c) shows the pressure-volume response for the same sinusoidal pore model in Fig. 3(a) but connected to a 0.2-m-long soft tube (model parameters in figure caption). As the meniscus moves towards the pore constriction, the pressure $\Delta P(x)$ decreases and the soft tube shrinks. After the pore constriction, the pressure increases and the soft tube expands (dashed blue line). The combined pressure-volume relationship $\Delta P-V_{\mathrm{sys}}$ (solid blue line) resembles the entrapped gas bubble case and exhibits a multivalued response. Once again, the meniscus jumps suddenly to a new position as the interface recedes and pressure shifts from the lower to the upper branch. Clearly, changes in pore volume will be more pronounced in more compliant matrices, e.g., soils versus rocks.

\section{Mechanism 3: Interacting menisci}

Interconnected menisci interact with each other through the fluid pressure, and reach the same Laplacian curvature under equilibrium conditions. The pressure decreases as the invading meniscus crosses the widest pore throat, and all 
(a) Stiff liquid in single, rigid pore

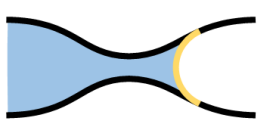

(b) "Soft" liquid (gas bubbles)

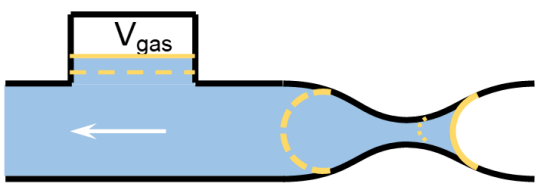

(c) "Soft" pore

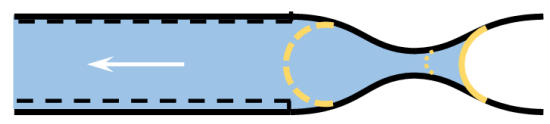

(d) Interacting menisci
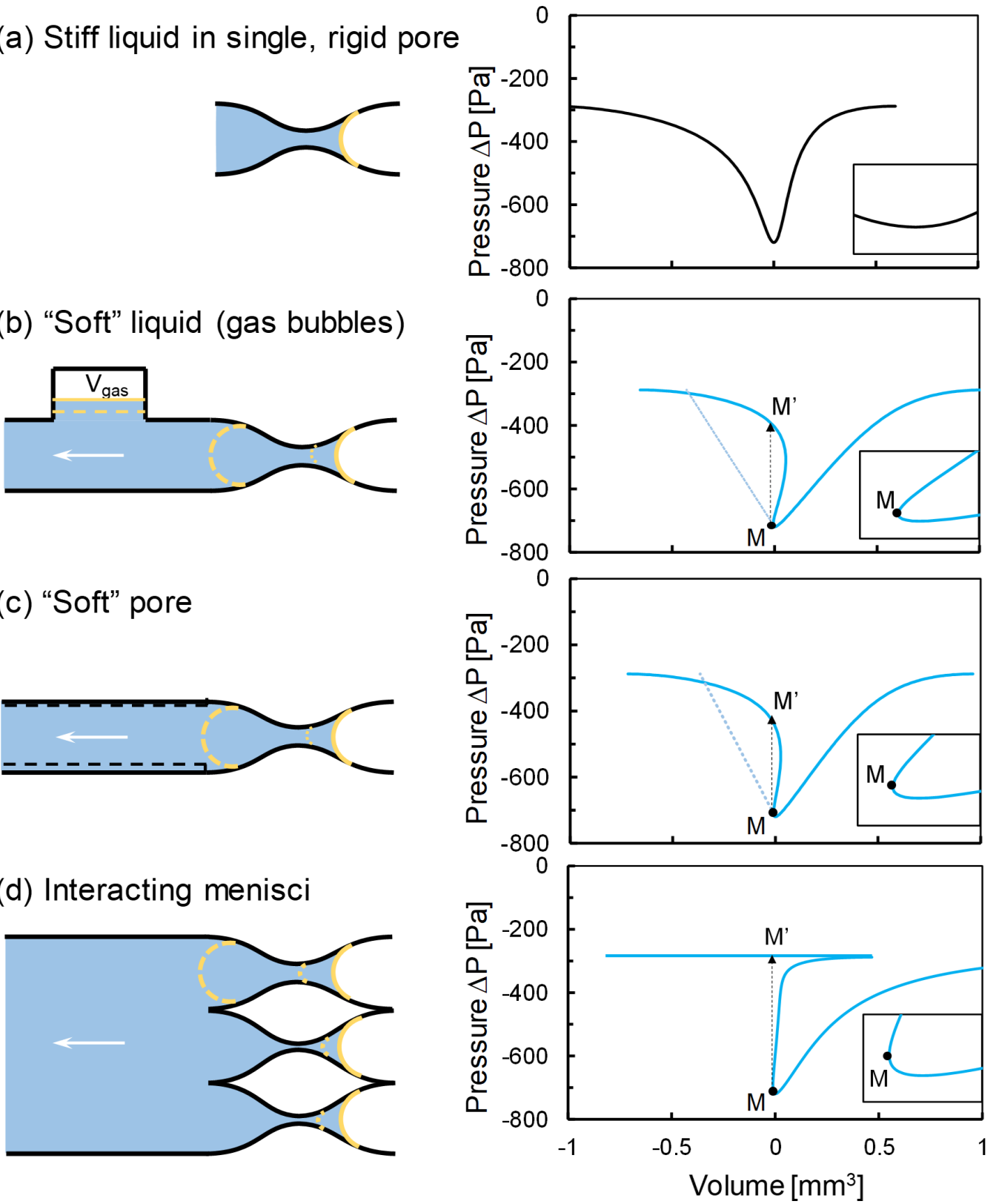

FIG. 3. Pore scale mechanisms for Haines jumps. (a) A sinusoidal pore ( $L=4 \mathrm{~mm}, r_{1}=0.5 \mathrm{~mm}$, and $\left.r_{2}=0.2 \mathrm{~mm}\right)$ with no gas bubbles, rigid matrix, and no interconnected menisci. (b) Mechanism 1: entrapped gas bubble. Case parameters: gas volume $V_{0}=0.1 \mathrm{ml}$, atmospheric pressure $P_{0}=101 \mathrm{kPa}$. (c) Mechanism 2: deformable solid matrix. Case parameters: $G=1.7 \mathrm{MPa}$, tube length $L_{\text {tube }}=0.2 \mathrm{~m}$. (d) Mechanism 3: interacting menisci. Case parameters: capillary opening radius $r_{1}=0.5 \mathrm{~mm}$, radius of wider pore constriction $r_{2}=0.2 \mathrm{~mm}$, radius of narrower pore constriction $r_{3}=0.18 \mathrm{~mm}$, pore constriction length $L=4 \mathrm{~mm}$. All cases: surface tension $\gamma=0.072 \mathrm{~N} / \mathrm{m}$ and contact angle $\theta=0^{\circ}$. Solid blue line: $\Delta P-V_{\text {sys }}$ curve, dashed line: $\Delta P-V_{\mathrm{el}}$ curve.

other menisci relax moving back and away from their pore constrictions. Every pore $i$ has its own $\Delta P-V_{\text {pore }}$ relationship:

$$
V_{\text {pore }}^{i}=f^{i}(\Delta P) .
$$

In this case, the "elastic" volumetric change $V_{\mathrm{el}}$ associated to the largest pore $m$ is the sum of the volume changes at all other interconnected pore constrictions for the same pressure change:

$$
V_{\mathrm{el}}=-\sum_{i \neq m} V_{\mathrm{pore}}^{i}
$$

Let us consider three interconnected pores where one pore throat $(r=0.20 \mathrm{~mm})$ is slightly larger than the other two $[r=0.18 \mathrm{~mm}$, Fig. 3(d)]. For clarity, both the matrix and the liquid are assumed infinitely rigid. The combined $\Delta P-V_{\text {sys }}$ curve is multivalued and the meniscus jumps to a new position with a corresponding jump in pressure (note: the $\Delta P-V_{\text {sys }}$ response shifts to the right as the number of interconnected pores $n$ increases). Clearly, interactions between interconnected menisci produce an equivalent soft system similar to an entrapped gas bubble and a compliant matrix. 
(a)
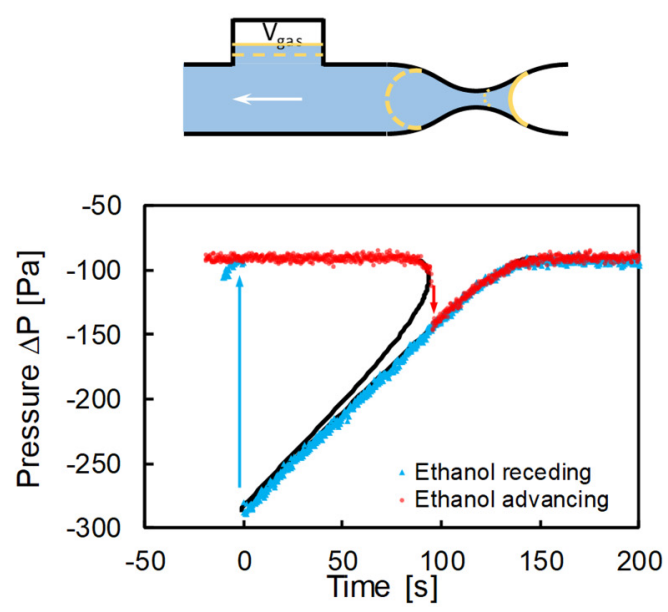

(b)
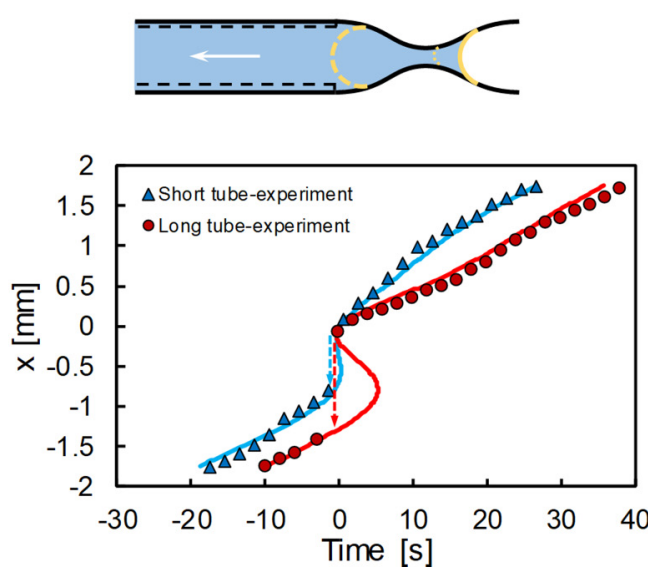

FIG. 4. Comparison between experimental measurements (red dots and blue triangles) in capillary tubes and model predictions (solid lines). (a) Haines jumps during advancing (red) and receding (blue) tests caused by interactions between menisci and entrapped gas bubbles. Model parameters: surface tension $\gamma=0.024 \mathrm{~N} / \mathrm{m}$ (ethanol-air), contact angle $\theta=22^{\circ}$, atmospheric pressure $P_{0}=101 \mathrm{kPa}$, volume of the entrapped gas bubble $V_{0}=1.48 \mathrm{ml}$ (inferred), capillary radius $r_{1}=0.48 \mathrm{~mm}$, pore constriction radius $r_{2}=0.16 \mathrm{~mm}$, pore constriction length $L=3.6 \mathrm{~mm}$. (b) Haines jumps caused by interactions between menisci and the plastic tubing. Model parameters: surface tension $\gamma=0.072$ $\mathrm{N} / \mathrm{m}$ (water-air), contact angle $\theta=27^{\circ}$, elastic modulus of Tygon tube $G=2 \mathrm{MPa}$, short tube length $L_{\text {tube }}=16.0 \mathrm{~cm}$ (blue), long tube length $L_{\text {tube }}=34.4 \mathrm{~cm}$ (red), capillary radius $r_{1}=0.48 \mathrm{~mm}$, pore constriction radius $r_{2}=0.16 \mathrm{~mm}$, pore constriction length $L=3.6 \mathrm{~mm}$.

\section{EXPERIMENTAL STUDY}

The complementary experimental study reported next explores Haines jumps by recording the fluid pressure and the interface position during immiscible displacement tests in pore models. We analyze experimental results within the theoretical framework described above.

\section{A. Experimental setup}

We test two pore systems: a capillary tube with a pore constriction and a microfluidic chip with interconnected pores.

Capillary tube. The borosilicate glass capillary tube $\left(2 r_{1}=\right.$ $0.97 \mathrm{~mm}$ ) has a constriction to simulate a sinusoidal pore throat $\left(2 r_{2}=0.32 \mathrm{~mm}, L=3.6 \mathrm{~mm}\right)$.

Microfluidic chip. The polydimethylsiloxane (PDMS) microfluidic chip is fabricated using standard soft lithography [23]. The microfluidic chip has three connected pores with the same opening width $w=0.5 \mathrm{~mm}$. One of the pore throats is slightly larger than the other two [i.e., $w_{1}=0.12 \mathrm{~mm}$, $w_{2}=w_{3}=0.1 \mathrm{~mm}$, Fig. 3(c)]. All channels are $0.1 \mathrm{~mm}$ deep.

Complementary devices. The experimental setup is similar to the setup in [15]. We use a syringe pump (Braintree Scientific Inc., BS-8000) to control the flow rate while we advance or recede the fluid interface across the pore model. The pressure transducer (Omega, PX40-50BHG5V, $\pm 6.7 \mathrm{kPa}$ ) mounted next to the inlet measures the pressure change. We observe and record the interface configuration using an optical microscope (Zeiss, Stemi 2000-CS).

Setup. The capillary tube connects to the syringe pump through a short, stiff polyurethane tube $(L=3 \mathrm{~cm} ; G=56$ $\mathrm{MPa}$, computed from the durometer based on [24]) to minimize the matrix compliance and to study the effect of trapped gas bubbles. Then we replace the polyurethane tube by a soft Tygon tube ( $G=2 \mathrm{MPa}$ ) of varying lengths to simulate a deformable porous matrix. The microfluidic chip connects to the syringe pump using stiff $1 / 16-i n$. ID Teflon tubing. De-ionized water (Milli-Q) and ethanol (VWR) are used as wetting liquids.

\section{B. Results}

Entrapped gas bubble. Figure 4(a) shows the pressure signature during a receding test (red dots) and an advancing test (blue triangles) conducted with ethanol and air, at an imposed injection rate of $q=80 \mu \mathrm{l} / \mathrm{h}$ (note: this flow rate would cause an average pore velocity of $3 \times 10^{-3} \mathrm{~cm} / \mathrm{s}$ in rigid pores of the same geometry). A gas bubble is introduced next to the pressure transducer. The analytical model (solid line) accurately predicts the position and amplitude of the Haines jump in both receding and advancing tests. Note that jumps happen at different positions during receding and advancing tests, resulting in distinct pressure-volume responses (see comprehensive dataset in [15]).

Deformable solid matrix. Figure 4(b) shows the position of the meniscus as a function of time in receding tests with a water-air system at an injection rate of $q=100 \mu \mathrm{l} / \mathrm{h}$. Data correspond to two system compliances controlled by the length of the Tygon tube ( $L_{\text {tube }}=16 \mathrm{~cm}$ in the short tube experiment and $L_{\text {tube }}=34.4 \mathrm{~cm}$ in the long tube experiment). Changes of the tube length produce different displacement results. Model predictions match experimental measurements.

Interacting menisci. Figure 5 displays the movement of ethanol-air menisci as ethanol is withdrawn across the pore throats in the PDMS microfluidic chip. All menisci move backwards at the beginning [Fig. 5(b)]. Then menisci stop receding across narrower pore constrictions [Fig. 5(c)]. One meniscus jumps across the largest pore throat, and the interconnected menisci relax and move back away from their pore 

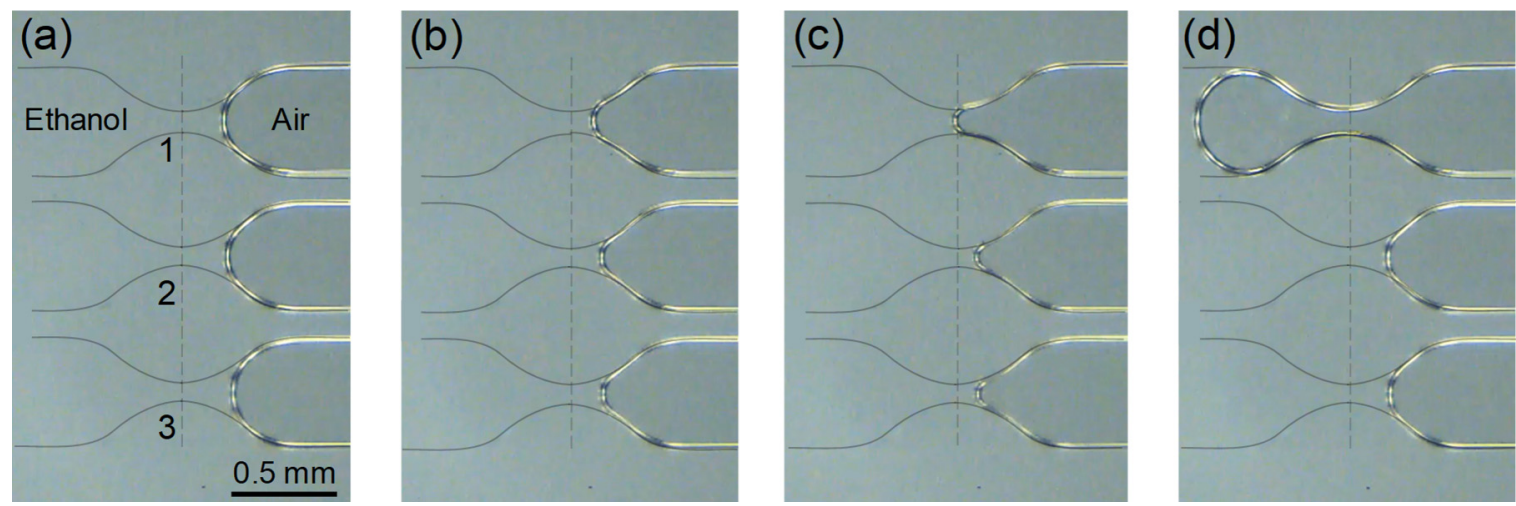

FIG. 5. Haines jumps caused by interacting menisci in a microfluidic chip.

constrictions [Fig. 5(d)]. These observations agree well with the mechanism of interacting menisci described above.

\section{DISCUSSION}

Analyses and experimental results presented above demonstrate that elastic and capillary interactions in porous media can result in Haines instabilities. In particular, we expect a Haines jump when the system response $\Delta P-V_{\text {sys }}$ is multivalued in capillary pressure $\Delta P$ for a specify displaced volume $V_{\text {sys }}$. A similar unstable response emerges in elastocapillary coalescence studies $[25,26]$. We can define a dimensionless elastocapillary number in terms of the system stiffness $k$ $\left(\mathrm{Pa} / \mathrm{m}^{3}\right)$ and capillarity for a given pore geometry (note: approximation for a sinusoidal pore with a small face angle $\alpha$-Fig. 2(a)—and linear elastic stiffness):

$$
N_{\mathrm{ec}}=\frac{k}{2 \gamma \cos (\theta-\alpha)} \frac{\pi r^{4}}{\sin \alpha} .
$$

A system with elastocapillary number $N_{\mathrm{ec}}<1$ is prone to Haines instabilities. The experimentally verified model and the proposed elastocapillary number allow us to predict the effects of different factors on the occurrence of Haines jumps.

\section{A. Effect of pore geometry}

The pressure change across a pore constriction increases as the radius of the pore constriction $r_{2}$ decreases relative to the pore size $r_{1}$. This magnifies the soft-system response, and increases the tendency to Haines jump. Figure 6(a) shows pressure-volume $\Delta P-V_{\text {sys }}$ response curves for pore models with $r_{2} / r_{1}=0.2,0.3$, and 0.4 (fixed $r_{1}=0.5 \mathrm{~mm}$ ) and trapped gas volume $V_{0}=0.05 \mathrm{ml}$. Haines jumps happen when pressure signatures are multivalued as in the $r_{2} / r_{1}=0.2$ and 0.3 cases; conversely, there is no instability in the $r_{2} / r_{1}=0.4$ case.

The meniscus may jump through more than one pore throat following a Haines instability. For example, the meniscus in Fig. 6(b) jumps at the pore throat 1 , drains pore 2 immediately, and settles at the pore throat $3\left(V_{0}=0.3 \mathrm{ml}\right.$; all other parameters are the same as in Fig. 6(a)]. This implies that a jump can drain the fluid in large pores and disturb internal fluid connectivity.

\section{B. Effect of surface tension and contact angle}

A lower surface tension creates smaller changes in pressure $\Delta P$ [Eq. (1)] and lessens the elastic volumetric response $V_{\text {el }}$. Indeed, we observe Haines jumps when air displaces water $\left(\gamma_{\text {air-water }}=0.072 \mathrm{~N} / \mathrm{m}\right)$, but jumps do not happen when air displaces alcohol in the same capillary $\left(\gamma_{\text {air-alcohol }}=\right.$ $0.024 \mathrm{~N} / \mathrm{m})$.

The contact angle plays a more profound role, as it affects both the amplitude and the shape of the $\Delta P-V_{\text {pore }}$ response $[15,27]$. When the contact angle is nonzero, the maximum meniscus curvature occurs after the meniscus passes the reference position at the constriction. Furthermore, the capillary pressure may be highest before the meniscus reaches the constriction. Figure 6(c) shows the effect of the contact angle on the $\Delta P-V_{\text {sys }}$ response for a liquid with entrapped air $\left(V_{0}=\right.$ $0.1 \mathrm{ml})$. Haines jumps occur for $\theta=0^{\circ}, 30^{\circ}$, and $60^{\circ}$, but there is no instability for $\theta=90^{\circ}$. The position of the jump and the pressure signature change with the contact angle and are distinct in advancing and receding tests.

Lastly, two instabilities may take place in a single pore across a pore throat if the system is soft enough. The case of a large gas bubble is plotted in Fig. 6(d): The two instabilities correspond to two sudden pressure changes in the pressure-time signature in an advancing test. Once again, this prediction is consistent with experimental observations (see [15]).

\section{Implications}

The above analyses and experimental results show that the number of interconnected pores $n$, pore constriction geometry $r_{2} / r_{1}$, and contact angle $\theta$ play a crucial role in predicting the occurrence of Haines jumps in porous media. High displacement efficiency can be achieved with intermediate wetting conditions when Haines jumps are minimized (see examples in $[7,28]$ ). While we are not able to effectively modify the pore geometry in natural porous media, we can alter wettability and the mechanical properties of interfaces with the addition of surfactants or nanoparticles.

Capillary effects can be significant in laboratory microfluidic systems due to the small pore sizes. Moreover, peripheral components can be very soft (e.g., entrapped gas, highly elastic PDMS and tubes). Therefore, laboratory studies with 
(a) Effect of pore geometry

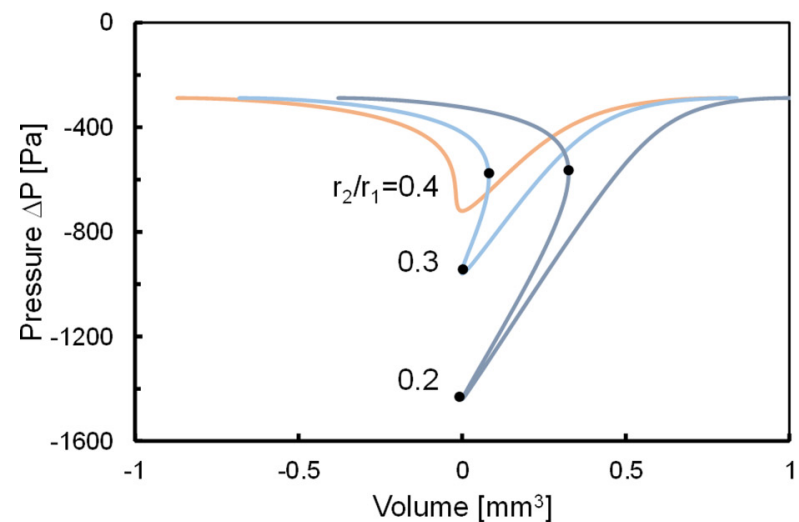

(c) Effect of contact angle

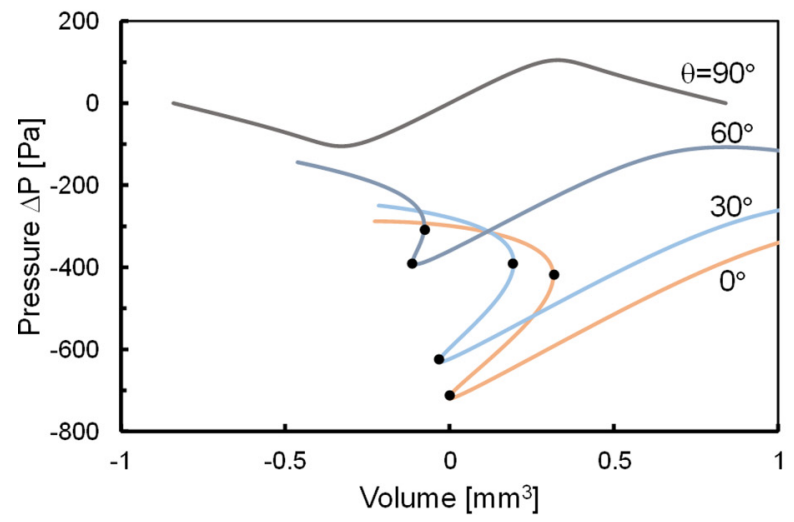

(b) Pores in series

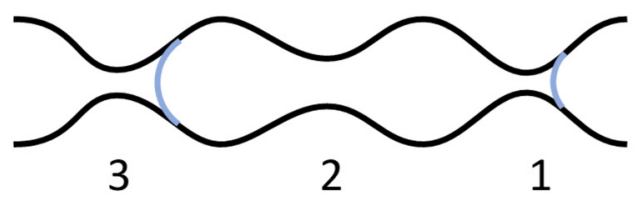

(d) Second instabilities

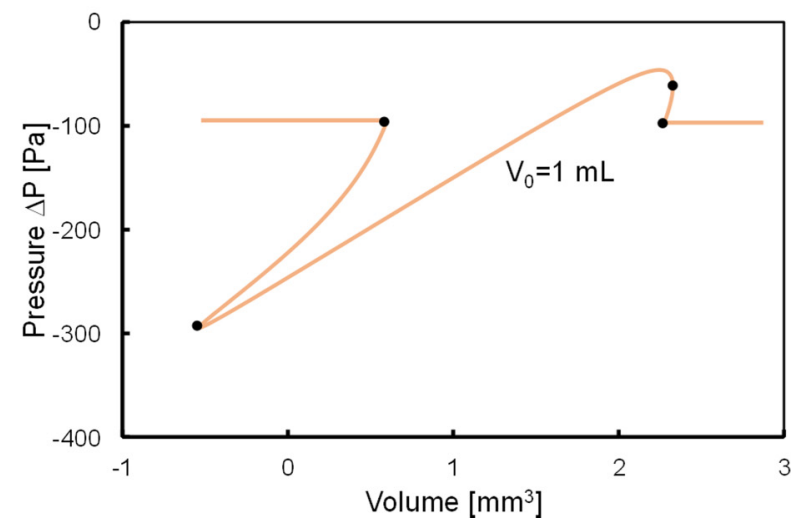

FIG. 6. Factors affecting Haines jumps. (a) Pressure-volume curves for different pore throat size to pore opening ratios $r_{2} / r_{1}$. Case parameters: gas volume $V_{0}=0.05 \mathrm{ml}$, surface tension $\gamma=0.072 \mathrm{~N} / \mathrm{m}$, contact angle $\theta=0^{\circ}$, atmospheric pressure $P_{0}=101 \mathrm{kPa}$, capillary opening radius $r_{1}=0.5 \mathrm{~mm}$, pore constriction length $L=4 \mathrm{~mm}$. (b) The meniscus jumps through more than one pore throat following a Haines instability in a series of pores. Radius of pore constriction $1 r_{2}=0.1 \mathrm{~mm}$, radius of pore constriction $2 r_{2}=0.2 \mathrm{~mm}$, radius of pore constriction $3 r_{2}=0.15 \mathrm{~mm}$. (c) Pressure-volume curves at different contact angles. (d) The pressure-volume curve for a system with a large gas bubble shows the occurrence of the secondary instability. Case parameters: surface tension $\gamma=0.072 \mathrm{~N} / \mathrm{m}$, contact angle $\theta=60^{\circ}$, atmospheric pressure $P_{0}=101 \mathrm{kPa}$, capillary opening radius $r_{1}=0.5 \mathrm{~mm}$, pore constriction radius $r_{2}=0.2 \mathrm{~mm}$, pore constriction length $L=4 \mathrm{~mm}$.

microfluidics should carefully consider the effects of "softsystem instabilities" on the observed flow behavior.

\section{CONCLUSIONS}

This study hypothesized and verified that Haines jumps take place in soft systems and involve a sudden change in pressure and fluid redistribution. We analyzed and tested three soft systems: entrapped gas bubble, compliant solid matrix, and interconnecting menisci. Equivalent conditions exist in both laboratory experiments and natural systems.

Haines jumps are more likely to occur in porous systems with pronounced pore constriction ratio $r_{2} / r_{1}$, small contact angles, and higher interfacial tension.
Matrix compliance, saturation, and pore geometry are predefined in natural systems. Still, Haines jumps can be controlled by changing the contact angle and/or interfacial properties (e.g., with the use of surfactants or nanoparticles). Laboratory microfluidic studies should consider the effects of entrapped gas bubbles and peripheral components compliance in the analysis of multiphase displacement test data.

\section{ACKNOWLEDGMENT}

Support for this research was provided by King Abdullah University of Science and Technology.
[1] J. C. Santamarina and Z. Sun, Mixed fluid conditions: Capillary phenomena, in Poromechanics VI, edited by M. Vandamme, P. Dangla, J. Pereira, and S. Ghabezloo (American Society of Civil Engineers, Reston, VA, 2017), pp. 70-89.
[2] W. B. Haines, J. Agric. Sci. 20, 97 (1930).

[3] N. R. Morrow, Ind. Eng. Chem. 62, 32 (1970).

[4] K. Singh, H. Scholl, M. Brinkmann, M. Di Michiel, M. Scheel, S. Herminghaus, and R. Seemann, Sci. Rep. 7, 444 (2017). 
[5] R. Holtzman and E. Segre, Phys. Rev. Lett. 115, 164501 (2015).

[6] S. Schlüter, S. Berg, M. Rücker, R. T. Armstrong, H. J. Vogel, R. Hilfer, and D. Wildenschild, Water Resour. Res. 52, 2194 (2016).

[7] B. Zhao, C. W. MacMinn, and R. Juanes, Proc. Natl. Acad. Sci. USA 113, 10251 (2016).

[8] J. C. Melrose, Can. J. Chem. Eng. 48, 638 (1970).

[9] J. C. Melrose, J. Can. Pet. Technol. 13, 54 (1974).

[10] R. M. Giordano and J. C. Slattery, J. Colloid Interface Sci. 92, 13 (1982).

[11] A. C. Payatakes, Annu. Rev. Fluid Mech. 14, 365 (1982).

[12] K. K. Mohanty, H. T. Davis, and L. E. Scriven, SPE Reservoir Eng. 2, 113 (1987).

[13] R. Sharma and D. S. Ross, J. Chem. Soc., Faraday Trans. 87, 619 (1991).

[14] W. W. Liou, Y. Peng, and P. E. Parker, J. Colloid Interface Sci. 333, 389 (2009).

[15] J. Jang, Z. Sun, and J. C. Santamarina, Water Resour. Res. 52, 9586 (2016).

[16] F. Moebius and D. Or, J. Colloid Interface Sci. 377, 406 (2012).

[17] R. T. Armstrong and S. Berg, Phys. Rev. E 88, 043010 (2013).
[18] Y. Edery, S. Berg, and D. Weitz, Phys. Rev. Lett. 120, 028005 (2018).

[19] R. Hilfer, R. T. Armstrong, S. Berg, A. Georgiadis, and H. Ott, Phys. Rev. E 92, 063023 (2015).

[20] R. Hilfer and P. E. Øren, Transp. Porous Media 22, 53 (1996).

[21] L. Liggieri, F. Ravera, and A. Passerone, J. Colloid Interface Sci. 140, 436 (1990).

[22] H. S. Yu, Cavity Expansion Methods in Geomechanics (Springer Science+Business Media, Berlin, 2000).

[23] S. Sia and G. M. Whitesides, Electrophoresis 24, 3563 (2003).

[24] A. Mix and A. Giacomin, J. Test. Eval. 39, 696 (2011).

[25] C. H. Mastrangelo and C. H. Hsu, J. Microelectromech. Syst. 2, 33 (1993).

[26] C. Duprat and H. A. Stone, Elastocapillarity, in Fluid-Structure Interactions in Low-Reynolds-Number Flows (The Royal Society of Chemistry, Cambridge, UK, 2016).

[27] G. Mason and N. R. Morrow, J. Colloid Interface Sci. 168, 130 (1994).

[28] M. Trojer, M. L. Szulczewski, and R. Juanes, Phys. Rev. Appl. 3, 054008 (2015). 